$\begin{array}{lll}\Omega & \equiv & \text { Dept of Child and Mother } \\ \text { Fernando M. de } & \text { pediatria@fmdebenedictis.it } \\ \text { Benedictis } & \text { Health, Salesi Children's } & \\ \text { Hospital, Ancona, Italy } & \end{array}$

\title{
GPs Meet Rare Lung Disorders Task Force factsheet: primary ciliary dyskinesia
}

\section{Definition}

Primary ciliary dyskinesia (PCD) is a rare, autosomal recessive disease of abnormalities of ciliary structure and function. The result is impaired mucociliary clearance, causing a variety of respiratory symptoms, and likely progression to bronchiectasis in most cases. Situs anomalies are present in nearly $50 \%$ of cases.

\section{Prevalence}

The prevalence of $P C D$ is thought to be around 1 in 10,000-15,000, but is very difficult to estimate accurately. This is due partly to difficulties in making the diagnosis, as a consequence of the large variation in clinical phenotype.

\section{Clinical manifestations}

Children with PCD often have a clinical history of lower airway disease, manifested in a chronic wet-sounding cough and, occasionally, wheeze or shortness of breath. In addition, virtually all subjects show evidence of chronic upper airway symptoms, such as chronic rhinitis (nasal discharge, episodic facial pain and anosmia). Ear symptoms (recurrent otitis media and glue ear) are a frequent complication that can require repeated courses of antibiotics. In table 1, the clinical aspects of PCD are displayed by various age groups. PCD is likely to include a small number of milder phenotypes that may be manifest in subtle or no apparent structural defects and ciliary dysfunction

\section{Diagnosis}

The diagnosis of PCD requires the presence of the characteristic clinical phenotype and either specific ultrastructural ciliary defects identified by transmission electron microscopy or evidence of abnormal ciliary function. Screening tests (nasal nitric oxide measurement, saccarin test) may precede formal ciliary function analysis, thereby reducing the number of referrals for ciliary analysis. A positive family history of PCD is an indication for performing diagnostics. Siblings of probands should also have PCD excluded. Several genes have been identified to date for autosomal recessive PCD variants. Genetic analyses may help to assess the carrier status of family members and provide tools for informed reproductive choices, although this is currently possible only for a minority of families. They may also become more important diagnostically since about $35 \%$ of PCD patients carry gene mutations.

\section{Referral}

Diagnosis should be confirmed in a specialist centre, as diagnostic analysis and interpretation are difficult.
CrossMark

$\leftarrow$ click for updates 
Table 1. Symptoms and findings of PCD according to age group

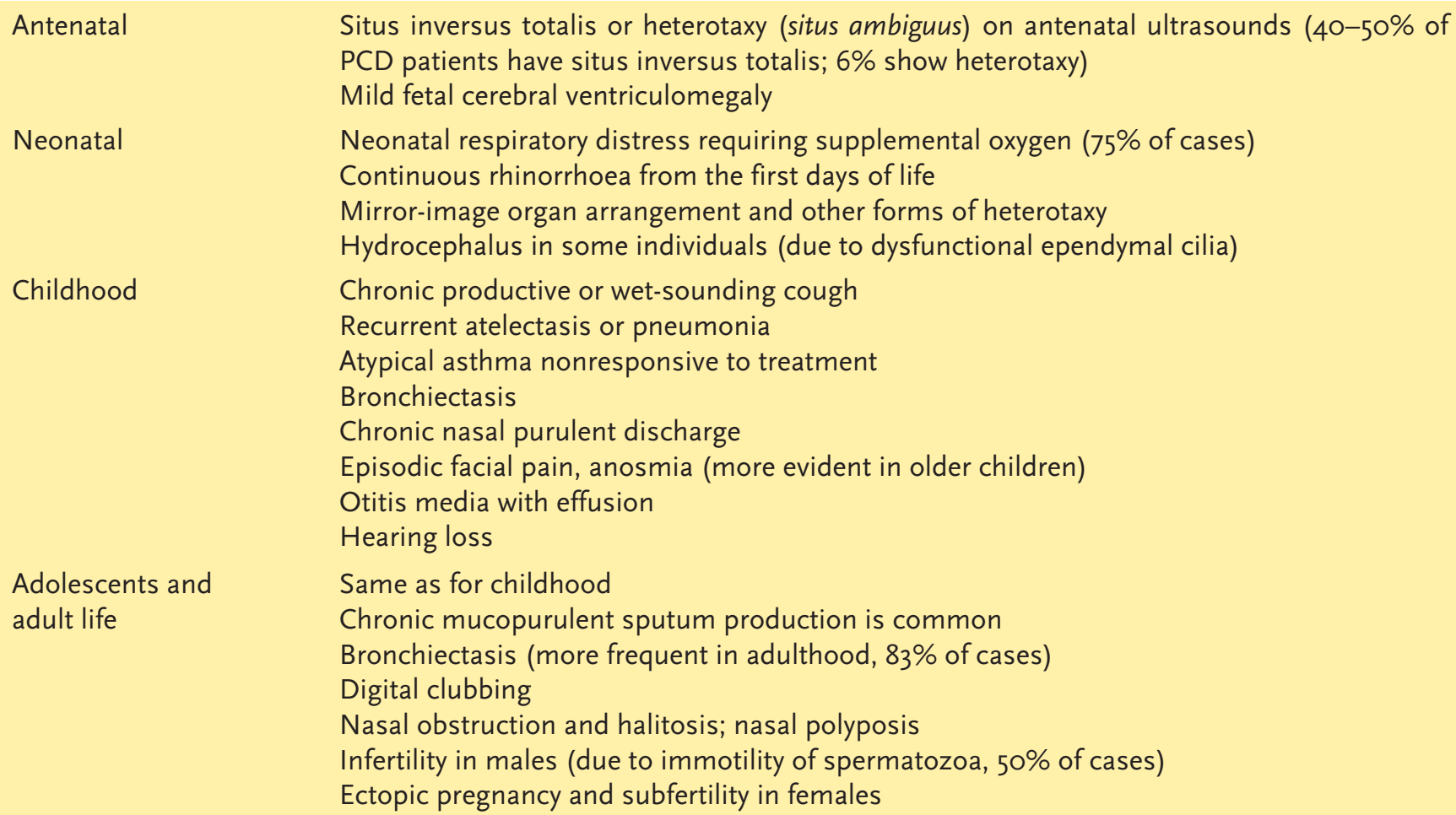

Neonata

Neonatal respiratory distress requiring supplemental oxygen ( $75 \%$ of cases)

Continuous rhinorrhoea from the first days of life

Mirror-image organ arrangement and other forms of heterotaxy

Hydrocephalus in some individuals (due to dysfunctional ependymal cilia)

Childhood

Chronic productive or wet-sounding cough

Recurrent atelectasis or pneumonia

Atypical asthma nonresponsive to treatment

Bronchiectasis

Chronic nasal purulent discharge

Episodic facial pain, anosmia (more evident in older children)

Otitis media with effusion

Hearing loss

Adolescents and adult life

Same as for childhood

Chronic mucopurulent sputum production is common

Bronchiectasis (more frequent in adulthood, $83 \%$ of cases)

Digital clubbing

Nasal obstruction and halitosis; nasal polyposis

Infertility in males (due to immotility of spermatozoa, $50 \%$ of cases)

Ectopic pregnancy and subfertility in females

Modified from ERS Task Force, Eur Respir J 2009; 34: 1264-1276.

\section{Treatment and follow-up}

As with all chronic respiratory diseases, the aim of therapy for PCD is to restore or maintain normal lung function as far as is possible, based on early detection and vigorous treatment of complications. Management of PCD involves aggressive treatment of upper and lower airway infections by antibiotics, and airway clearance by combinations of physiotherapy and physical exercise.

By analogy with cystic fibrosis (CF), PCD patients should be managed in specialised centres, in which they have regular access to respiratory paediatricians, audiology, ENT surgeons and respiratory physiotherapists.

\section{Key messages}

- Presentation in PCD is often non-specific, or has features typical of many childhood illnesses

- Think about PCD if your patient has chronic symptoms of both upper and lower airway infection and situs inversus viscerum

- $\quad$ PCD is an important cause of progressive lung disease and our key aim is to establish an early diagnosis and institute treatment strategies prior to the onset of irreversible lung damage.

- Refer the patient to a centre with experience in performing ultrastructural and functional studies of cilia

- Follow your patient in collaboration with the reference centre 


\section{Case report}

A 7-year-old boy was referred to a paediatric outpatient clinic for asthma resistant to treatment. He had a history of nasal obstruction with chronic mucopurulent discharge and chronic cough since the first months of life. He also presented with recurrent episodes of otitis media with secretion during infancy and two episodes of pneumonia during his preschool years. No overt episodes of wheezing were described. At the age of 6 years, a skin prick test showed positivity to house dust mites and alternaria. The diagnosis of allergic rhinitis and asthma was made, and treatment with inhaled corticosteroids and antihistamines was given for months, with no success.

At physical examination, situs inversus viscerum with dextrocardia was revealed. Spirometry was normal. Electron microscopy revealed ultrastructural ciliary defects in $100 \%$ of cilia obtained by nasal brushing. The diagnosis of primary cilia dyskinesia was made and proper management was planned.

\section{Further reading}

1. Barbato A, Frisher T, Kuehni CE, et al. Primary ciliary dyskinesia: a consensus statement on diagnostic and treatment approaches in children. Eur Resp J 2009; 34: 1264-1276.

2. Strippoli MP, Frischer T, Barbato A, et al. ERS Task Force on Primary Ciliary Dyskinesia in Children. Management of primary ciliary dyskinesia in European children: recommendations and clinical practice. Eur Respir J 2012; 39: 1482-1491.

3. www.pcdfoundation.org. The foundation of Primary Ciliary Diskinesia syndrome.

4. www.orpha.net. The portal for Rare Diseases and orphan drugs. 